\title{
Comparative genomic analysis and characteristics of NCCP15740, the major type of enterotoxigenic Escherichia coli in Korea
}

\author{
Taesoo Kwon ${ }^{1 \dagger}$, Si-yun Chung ${ }^{1 \dagger}$, Young-Hee Jung ${ }^{2}$, Su-Jin Jung ${ }^{3}$, Sang-Gyun Roh ${ }^{4}$, Je-Seop Park ${ }^{5}$, \\ Cheorl-Ho Kim ${ }^{6}$, Won Kim ${ }^{1}$, Young-Seok Bak ${ }^{4 \ddagger}$ and Seung-Hak Cho ${ }^{7^{*} \ddagger}$ (])
}

\begin{abstract}
Background: Enterotoxigenic Escherichia coli (ETEC) cause infectious diarrhea and diarrheal death. However, the genetic properties of pathogenic strains vary spatially and temporally, making prevention and treatment difficult. In this study, the genomic features of the major type of ETEC in Korea from 2003 to 2011 were examined by wholegenome sequencing of strain NCCP15740, and a comparative genomic analysis was performed with O6 reference strains.
\end{abstract}

Results: The assembled genome size of NCCP15740 was 4,795,873 bp with $50.54 \% \mathrm{G}+\mathrm{C}$ content. Using rapid annotation using subsystem technology analysis, we predicted 4492 ORFs and 17 RNA genes. NCCP15740 was investigated for enterotoxin genes, colonization factor (CF) genes, serotype, multilocus sequence typing (MLST) profiles, and classical and nonclassical virulence factors. NCCP15740 belonged to the O6:H16 serotype and possessed enterotoxin genes encoding heat-stable toxin (STh) and heat-labile toxin (LT); 87.5\% of the O6 serotype strains possessed both toxin types. NCCP15740 carried the colonization factors CS2 and CS3, whereas most O6 strains carried CS2-CS3-CS21 (79.2\%). NCCP15740 harbored fewer virulence factors (59.4\%) than the average observed in other O6 strains (62.0\%). Interestingly, NCCP15740 did not harbor any nonclassical virulence genes.

Conclusions: The major type of ETEC in Korea had the same MLST sequence type as that of isolates from the USA obtained in 2011 and 2014, but had different colonization factor types and virulence profiles. These results provide important information for the development of an ETEC vaccine candidate.

Keywords: Enterotoxigenic Escherichia coli O6, Whole-genome sequencing, Colonization factor genes, Enterotoxin, Multilocus sequence typing, Virulence

\section{Background}

Escherichia coli is a rod-shaped, gram-negative, facultative anaerobic and non sporulating bacterium belonging to the family Enterobacteriaceae. E. coli inhabits the intestines of all humans and animals. Most E. coli are harmless, but some induce various diseases; thus, the species is considered an opportunistic pathogen. E. coli

\footnotetext{
${ }^{*}$ Correspondence: skcho38@korea.kr

'Taesoo Kwon and Si-yun Chung contributed equally to this work

†Young-Seok Bak and Seung-Hak Cho contributed equally to this work

${ }^{7}$ Division of Enteric Diseases, Center for Infectious Diseases, Korea

National Institute of Health, Cheongju 363-951, Republic of Korea

Full list of author information is available at the end of the article
}

strains that cause diarrhea can be categorized into six groups according to virulence elements in the genome: enterotoxigenic (ETEC), enteropathogenic, nonsporulating, enteroaggregative, enteroinvasive, and diffusely adherent [1]. ETEC is a major cause of traveler's diarrhea and is responsible for 700,000 diarrhea-related deaths per year in young children of less than 5 years of age in developing countries $[2,3]$. Among the major virulence factors, two enterotoxins, i.e., a heat-labile toxin (LT) and a heat-stable toxin (ST), induce watery diarrhea in ETEC. The LT toxin is encoded by the eltAB gene. ST toxins are classified into two types, STh and STp; humanderived STh is encoded by est $A$, and porcine-derived STp 
is encoded by st1 [4]. In addition to serotyping, ETEC strains are classified by the combination of the $\mathrm{O}$ antigen of the lipopolysaccharide, $\mathrm{H}$ antigen of the flagellin, and $\mathrm{K}$ antigens. Although there are over 100 different $\mathrm{O}$ antigens and $34 \mathrm{H}$ antigens associated with ETEC $[5,6], \mathrm{O}$, O8, O25, O78, O128, and $\mathrm{O} 153$ and H7, H12, H16, H21, $\mathrm{H} 45$, and $\mathrm{H} 49$ are the most common, respectively [7]. In addition to enterotoxins, ETEC strains possess adhesive pili called colonization factors (CFs), which mediate adherence to the small intestinal wall. Over $30 \mathrm{CFs}$ have been described in human ETEC strains to date. The most prevalent CFs are CFA/I and CS1-CS6, and strains typically carry two or three CFs, such as CS1 + CS3, $\mathrm{CS} 2+\mathrm{CS} 3$, and CS5 + CS6.

In a previous study [8], 258 isolates from patients with diarrhea in Korea and 33 isolates from travelers visiting other Asian countries were analyzed, and two major sequence types were identified by multilocus sequence typing (MLST). In particular, ST171 $(\mathrm{n}=62)$ was identified as the most prevalent ETEC type in Korea, but ST949 $(\mathrm{n}=5)$ was the most frequent among inflow isolates. Although ST171 was a major MLST type of ETEC in Korea, the genomic characteristics, including enterotoxin genes, CF genes, and virulence factors, had not yet been investigated. In the present study, we selected one ST171 strain identified in this previous work, i.e., NCCP15740, isolated in 2010 from a patient with diarrhea, with serotype O6:H16, and performed whole-genome sequencing. We compared the genome of NCCP15740 with other whole-genome sequences of ETEC strains reported as O6:H16 isolates over a similar time period.

\section{Methods}

\section{Strains, isolation, and serotyping}

Escherichia coli NCCP15740 was isolated in 2010 from a patient with diarrhea and identified as a major MLST type (ST 171) of ETEC in Korea based on 24 isolates obtained from 2003 to 2011 [8]. Candidate colonies of NCCP15740 were identified based on phenotypes and biochemical properties using the API20E system (Biomerieux, Marcy l'Etoile, France). E. coli ATCC 25922 [9] was used as a reference strain to investigate the characteristics of NCCP15740. E. coli ATCC 25922 is an O6 serotype ETEC (O6:H1) reference strain. Moreover, we selected 19 E. coli O6 strains $(\mathrm{O} 6: \mathrm{H} 16)[10,11]$ as reference strains because they had the same serotype as NCCP15740. The 19 E. coli O6 strains were isolated in the USA from 2011 to 2014. From the comparison with the $19 E$. coli O6 strains, we expected that the evolutionary relationship with the strains identified from a similar period as NCCP15740 could be estimated. Two additional strains were used as reference strains: E. coli O6:H16:CFA/II str. B2C (traveler's diarrhea) [12] and E. coli O6:H16 str. 99-3165 (USA) [13].

\section{Library preparation and whole-genome sequencing}

A TruSeq sample preparation kit (Illumina, San Diego, CA, USA) was used to construct a sequencing library. Whole-genome sequencing of NCCP15740 was performed using the Illumina HiSeq 2000 platform (Theragen Etex Bio Institute, Suwon, Republic of Korea).

\section{Genome assembly and annotation}

High-quality reads were obtained by discarding reads with quality scores of less than Q20 and were assembled into scaffolds, using SOAPdenovo (version 1.05) [14]. Open reading frames were predicted and annotated by rapid annotation using subsystem technology (RAST, version 4.0) server [15]. In silico serotyping of NCCP15740 and other reference strains was performed using SerotypeFinder (version 1.1) [16]. MLST typing was also performed using the E. coli MLST database [17]. The genomic and phenotypic characteristics of NCCP15740 and the reference strains are summarized in Table 1.

\section{Phylogenetic analysis}

Multiple sequence alignments were obtained from wholegenome sequences of $24 \mathrm{E}$. coli strains and from seven MLST genes of the E. coli isolates, i.e. $a d k, f u m C, \operatorname{gyr} B$, $i c d, m d h$, purA, and $\operatorname{rec} A[18,19]$, using Mugsy (version 1.2.3) [20]. Approximate maximum-likelihood phylogenetic trees were generated using FastTree (version 2.1.7) [21] with the generalized time-reversible + CAT model [22]. The resulting trees were visualized using FigTree (version 1.3.1; http://tree.bio.ed.ac.uk/software/figtree/).

\section{Analysis of virulence factors}

To inspect virulence factor-encoding genes, BLAST searches of whole coding sequences (CDSs) were performed against the virulence factor database VFDB [23] adopting an e-value threshold of 1e-5. In addition, the BLAST Score Ratio (BSR) [24] was calculated to identify homologous virulence factor genes. A BSR threshold of at least 0.7 was used in this study.

\section{Quality assurance}

The genomic DNAs were purified from a pure culture of a single bacterial isolate of NCCP15740. Potential

\section{Table 1 Genomic features of NCCP15740}

\begin{tabular}{ll}
\hline Strain & NCCP15740 \\
\hline Genome (Mb) & 4.79 \\
\%GC & 50.54 \\
Total open reading frames & 4492 \\
tRNAs & 17 \\
rRNAs & 0 \\
\hline
\end{tabular}


contamination of the genomic libraries by other microorganisms was evaluated using a BLAST search against the nonredundant database.

\section{Results}

\section{General features}

Using the Illumina HiSeq 2000 platform, we generated a total of 548,710,000 bp paired-end reads (86.32-fold coverage). After quality control, $495 \mathrm{Mbp}$ of high-quality reads were de novo assembled into 156 scaffolds with a scaffold N50 of 87,362 bp. The NCCP15740 genome was $4,795,873$ bp in length (Fig. 1), and the G+C content was $50.54 \%$. Using the RAST server pipeline, 4492 putative coding sequences and 17 RNA genes were identified. The genomic properties of NCCP15740 are summarized in Table 1. According to in silico analysis, the NCCP15740 serotype was O6:H16.

\section{Phylogenetic analysis}

A whole-genome phylogeny was constructed from the alignments of the genomes of $24 \mathrm{E}$. coli isolates, and an MLST-based phylogeny was constructed from the alignments of seven MLST genes of the E. coli isolates (Fig. 2). The E. coli O6 strains had simple phylogenetic relationships, represented by three sequence types, according to both the whole-genome and MLST data. The wholegenome phylogeny showed that NCCP15740 belonged to a group of strains isolated in 2011 and was distinct from the majority of $\mathrm{O} 6$ strains, although it was isolated in 2010. In contrast to the whole-genome phylogeny, all isolates obtained in 2011 clustered in the same group in the MLST-based phylogeny. Only three 2011 isolates clustered with NCCP15740 in the whole-genome phylogeny, whereas 14 isolates obtained in 2011 formed a cluster in the MLST-based phylogeny. Based on MLST, the most

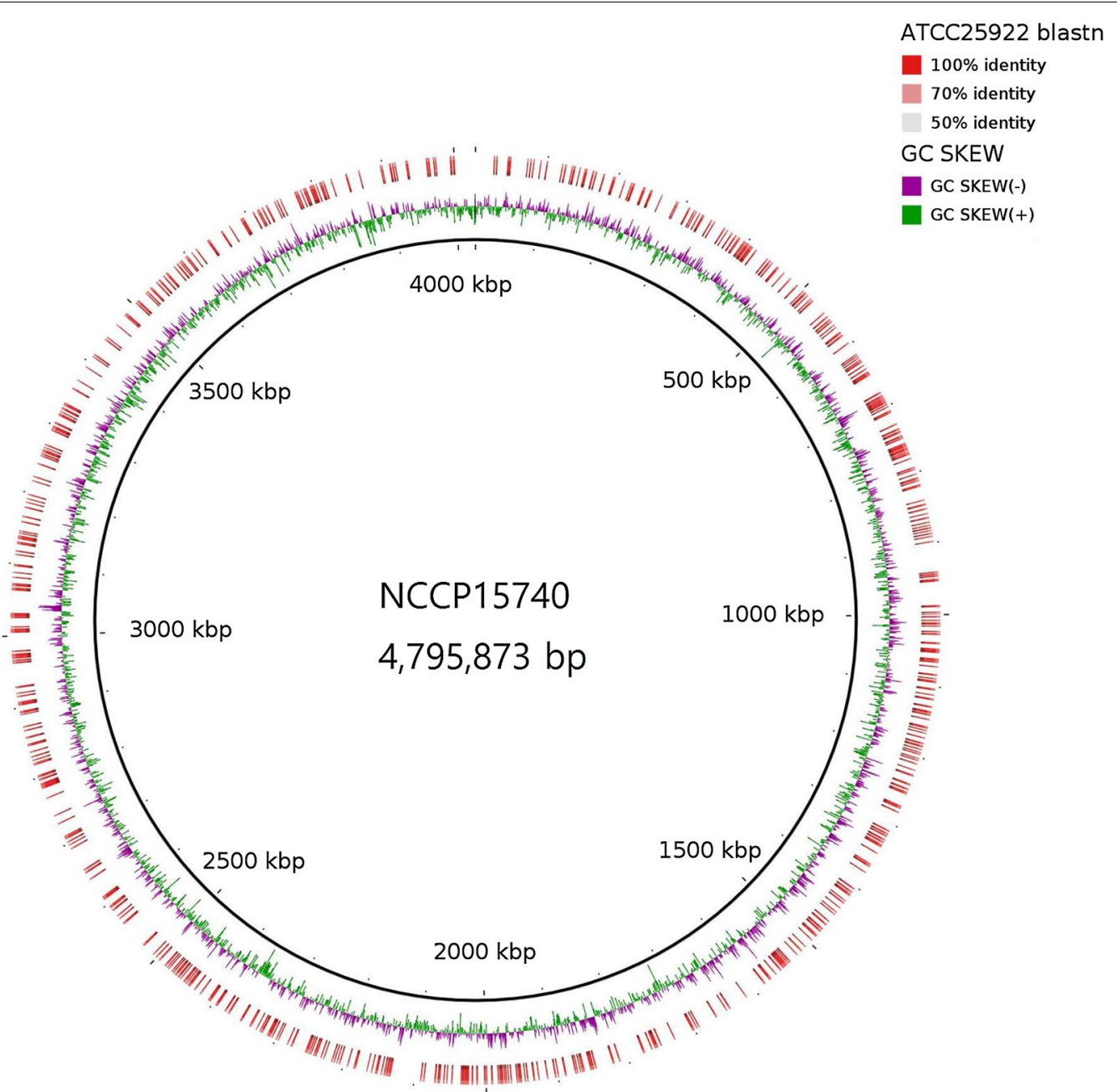

Fig. 1 Circular map of NCCP15740. The map was created using BLAST ring image generator (BRIG) version 0.95 

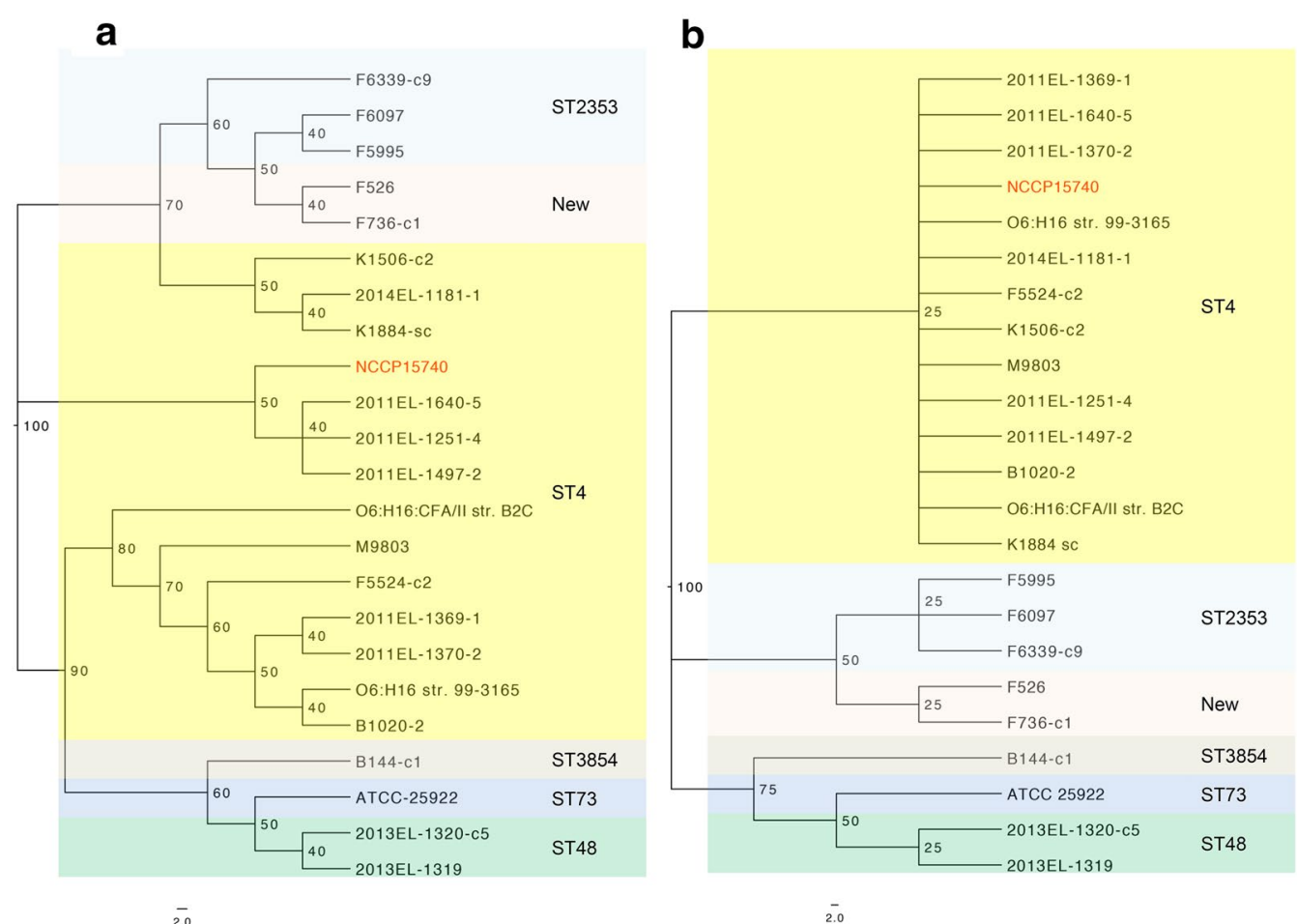

$\overline{2.0}$

Fig. 2 Phylogenetic tree of NCCP15740. a Whole-genome phylogeny, b MLST-based phylogeny. Evolutionary time is scaled by 100; lower values imply a relatively recent branching event. The scale bar indicates 2.0 substitutions per site. NCCP15740 (red) was placed in a single clade with 2011EL 1251-4, 2011EL 1497-2, and 2011EL 1640-5 in both the whole-genome phylogeny and the MLST phylogeny

prevalent sequence type was ST4 (62.5\%), followed by ST2353 (12.5\%). The sequence type of NCCP15740 was ST4.

\section{Identification of enterotoxins and colonization surface antigens}

We investigated the toxin types of NCCP15740 and reference strains. As shown in Table 2, consistent with the major type of Korean ETEC isolates, NCCP15740 carried both LT and ST enterotoxins, and the presence of both STh and LT was common in the ETEC O6 strains (21/24). Moreover, CS types were found in $87.5 \%$ of all isolates, including NCCP15740. Among the strains of O6 serotype in this study, CS2 + CS3 + CS21 was the most prevalent type, and four different CS types were found. CS21 was detected in $20(83.3 \%)$ of the 24 ETEC O6 strains, but was not detected in two (8.3\%) strains containing only STh and in one (4.1\%) strain containing both STh and LT, i.e., NCCP15740.

\section{Analysis of virulence factors}

To determine the causal mechanisms underlying the observed pathogenicity [8], we compared virulence factors in NCCP15740 with those of the reference strains (Additional file 1: Figure S1). The strains harbored 207 total virulence factors classified into 27 categories and 66 subcategories. NCCP15740 harbored 123 of the 207 virulence factors $(59.4 \%)$, which was fewer than the average number of virulence factors in the reference strains used in this study $(128 / 207,62.0 \%)$. Several virulence factors that were found in the majority of the O6 strains were not present in NCCP15740, including ibeB, etpA, cah, fimZ, tia, tuf, flgD, flgE, ipaH2.5, and aatC. Nonclassical virulence factors related to adherence, invasion, secretion, and iron acquisition are the main contributors to ETEC diarrhea [25]. Surprisingly, most of the nonclassical virulence factors that have been found in ETEC strains [25], including eat $A$, etpB, fyuA, leoA, and tibA, were not present in $\mathrm{O} 6$ strains. Only three nonclassical virulence factors were found in O6 strains, i.e., etpA (18 out of 24), irp2 (only in E. coli ATCC 25922), and tia (11 out of 24). However, none of the nonclassical virulence factors were found in NCCP15740.

\section{Discussion}

ETEC is responsible for 700,000 diarrhea-related deaths per year in young children of less than 5 years of age and is a main cause of traveler's diarrhea [3]. However, the type and relative proportions of ETEC enterotoxins differ depending on the geographical source. 
Table 2 Genomic features of NCCP15740 and other strains used in this study

\begin{tabular}{|c|c|c|c|c|c|c|c|}
\hline Strain & Genome (Mb) & MLST type & Serotype & Toxin type & CS type & $\begin{array}{l}\text { GenBank } \\
\text { accession }\end{array}$ & $\begin{array}{l}\text { GenBank } \\
\text { assembly }\end{array}$ \\
\hline E. coli NCCP15740 & 4.79 & ST4 & $\mathrm{O} 6: \mathrm{H} 16$ & $\mathrm{STh}+\mathrm{LT}$ & $\mathrm{CS} 2+\mathrm{CS} 3$ & MOED01 & GCA_001865725 \\
\hline E. coli ATCC25922 & 5.20 & ST73 & $\mathrm{O} 6: \mathrm{H} 1$ & None & None & CP009072 & GCA_000743255 \\
\hline E. coli O6:H16:CFA/II str. B2C & 5.02 & ST4 & $\mathrm{O} 6: \mathrm{H} 16$ & $\mathrm{STh}+\mathrm{LT}$ & $\mathrm{CS} 2+\mathrm{CS} 3+\mathrm{CS} 21$ & AUZS01 & GCA_000517245 \\
\hline E. coli O6:H16 str. 99-3165 & 4.75 & ST4 & $\mathrm{O} 6: \mathrm{H} 16$ & $\mathrm{STh}+\mathrm{LT}$ & $\mathrm{CS} 2+\mathrm{CS} 3+\mathrm{CS} 21$ & JHJW01 & GCA_000614435 \\
\hline E. coli strain $2011 \mathrm{EL}-1370-2$ & 4.81 & ST4 & $\mathrm{O} 6: \mathrm{H} 16$ & $\mathrm{STh}+\mathrm{LT}$ & $\mathrm{CS} 2+\mathrm{CS} 3+\mathrm{CS} 21$ & JPUT01 & GCA_000770035 \\
\hline E. coli strain 2011 EL-1369-1 & 4.80 & ST4 & $\mathrm{O} 6: \mathrm{H} 16$ & $\mathrm{STh}+\mathrm{LT}$ & $\mathrm{CS} 2+\mathrm{CS} 3+\mathrm{CS} 21$ & JPUS01 & GCA_000770045 \\
\hline E. coli strain 2011 EL-1497-2 & 4.97 & ST4 & $\mathrm{O} 6: \mathrm{H} 16$ & $\mathrm{STh}+\mathrm{LT}$ & $\mathrm{CS} 2+\mathrm{CS} 3+\mathrm{CS} 21$ & JPUU01 & GCA_000770055 \\
\hline E. coli strain 2013EL-1320-c5 & 4.71 & ST48 & $\mathrm{O} 6: \mathrm{H} 16$ & STh & None & JPUD01 & GCA_000770255 \\
\hline E. coli strain 2013 EL-1319 & 4.71 & ST48 & $\mathrm{O} 6: \mathrm{H} 16$ & STh & None & JPIF01 & GCA_000773435 \\
\hline E. coli strain F5995 & 5.17 & ST2353 & $\mathrm{O} 6: \mathrm{H} 16$ & $\mathrm{STh}+\mathrm{LT}$ & $\mathrm{CS} 2+\mathrm{CS} 3+\mathrm{CS} 21$ & JPXL01 & GCA_000773475 \\
\hline E. coli strain M9803 & 4.88 & ST4 & $\mathrm{O} 6: \mathrm{H} 16$ & $\mathrm{STh}+\mathrm{LT}$ & $\mathrm{CS} 2+\mathrm{CS} 3+\mathrm{CS} 21$ & JPXK01 & GCA_000773535 \\
\hline E. coli strain F6097 & 5.19 & ST2353 & $\mathrm{O} 6 \mathrm{H} 16$ & $\mathrm{STh}+\mathrm{LT}$ & $\mathrm{CS} 2+\mathrm{CS} 3+\mathrm{CS} 21$ & JPXJ01 & GCA_000773555 \\
\hline E. coli strain 2011 EL-1640-5 & 4.87 & ST4 & $\mathrm{O} 6 \mathrm{H} 16$ & $\mathrm{STh}+\mathrm{LT}$ & $\mathrm{CS} 2+\mathrm{CS} 3+\mathrm{CS} 21$ & JPXN01 & GCA_000773575 \\
\hline E. coli strain $2011 \mathrm{EL}-1251-4$ & 4.88 & ST4 & $\mathrm{O} 6 \mathrm{H} 16$ & $\mathrm{STh}+\mathrm{LT}$ & $\mathrm{CS} 2+\mathrm{CS} 3+\mathrm{CS} 21$ & JPXM01 & GCA_000773595 \\
\hline E. coli strain K1506-c2 & 4.94 & ST4 & O6:H16 & $\mathrm{STh}+\mathrm{LT}$ & $\mathrm{CS} 2+\mathrm{CS} 3+\mathrm{CS} 21$ & JYIB01 & GCA_000935345 \\
\hline E. coli strain F526 & 4.95 & New & $\mathrm{O} 6: \mathrm{H} 16$ & $\mathrm{STh}+\mathrm{LT}$ & $\mathrm{CS} 2+\mathrm{CS} 3+\mathrm{CS} 21$ & JYHX01 & GCA_000935365 \\
\hline E. coli strain 2014EL-1181-1 & 4.95 & ST4 & $\mathrm{O} 6: \mathrm{H} 16$ & $\mathrm{STh}+\mathrm{LT}$ & $\mathrm{CS} 2+\mathrm{CS} 3+\mathrm{CS} 21$ & JYIE01 & GCA_000935375 \\
\hline E. coli strain F736-c1 & 4.95 & New & $\mathrm{O} 6: \mathrm{H} 16$ & $\mathrm{STh}+\mathrm{LT}$ & $\mathrm{CS} 2+\mathrm{CS} 3+\mathrm{CS} 21$ & JYHY01 & GCA_000935425 \\
\hline E. coli strain B144-c1 & 4.90 & ST3854 & $\mathrm{O} 6: \mathrm{H} 16$ & $\mathrm{STh}+\mathrm{LT}$ & $\mathrm{CS} 2+\mathrm{CS} 3+\mathrm{CS} 21$ & JYID01 & GCA_000935435 \\
\hline E. coli strain K1884 sc & 4.99 & ST4 & O6:H16 & $\mathrm{STh}+\mathrm{LT}$ & $\mathrm{CS} 2+\mathrm{CS} 3+\mathrm{CS} 21$ & JYIC01 & GCA_000935445 \\
\hline E. coli strain B1020-2 & 4.77 & ST4 & $\mathrm{O} 6: \mathrm{H} 16$ & $\mathrm{STh}+\mathrm{LT}$ & $\mathrm{CS} 2+\mathrm{CS} 3+\mathrm{CS} 21$ & JYIF01 & GCA_000935515 \\
\hline E. coli strain F6339-c9 & 4.91 & ST2353 & O6:H16 & $\mathrm{STh}+\mathrm{LT}$ & $\mathrm{CS} 2+\mathrm{CS} 3+\mathrm{CS} 21$ & $\mathrm{JY} \mid \mathrm{H} 01$ & GCA_000935525 \\
\hline E. coli strain F5524-c2 & 4.88 & ST4 & $\mathrm{O} 6: \mathrm{H} 16$ & $\mathrm{STh}+\mathrm{LT}$ & $\mathrm{CS} 2+\mathrm{CS} 3+\mathrm{CS} 21$ & JYIG01 & GCA_000935555 \\
\hline
\end{tabular}

The enterotoxin types of Korean isolates from 2003 to 2011 were reported to be similar to those of isolates from Asia and the Middle East, but different from those of isolates from South America [8]. In this study, we investigated the characteristics of the major type of ETEC in Korea at the genomic level by sequencing an ST171 isolate, NCCP15740, and performing a comparative analysis with the genome sequences of other O6 strains. According to the whole-genome phylogeny, NCCP15740 belonged to one of the two groups of strains that were isolated in 2011, but belonged to the group that included the majority of O6 strains in the MLST-based phylogeny (Fig. 2). There are many genomic changes that determine the branch of a strain in a phylogenetic tree, including SNPs, insertions, deletions, prophages, and other insertion sequence elements. However, MLST genes are housekeeping genes and are more conserved than other genomic loci. Therefore, whole-genome-based phylogeny is more sensitive than MLST-based phylogeny, although it is more difficult to group strains with whole-genomebased phylogeny. Accordingly, it is necessary to select whole-genome- or MLST-based phylogeny according to the needs of the study design and aim. An MLST-base phylogeny is suitable for clustering strains according to their MLST type, whereas whole-genome phylogeny provides a better representation of the differences between strains than MLST-based phylogeny.

We investigated the toxin types of NCCP15740 and reference strains. Strains that only express LT are generally less pathogenic [26]. The NCCP15740 genome had genes encoding both STh and LT enterotoxin types. The presence of both STh and LT was common in O6 strains (21 out of 24).

The genomes of ETEC strains harbor genes encoding more than one type of CF [27]. According to a previous study [8], the CS3/CS21 genes are the most prevalent CF genes in Korean isolates, and CS3-CS21-CS1/PCF071 (15/64) and CS2-CS3-CS21 (13/64) are the most frequent CF genes in ST171. In contrast, NCCP15740 had CS2/ CS3 genes that were only observed in two ST171 isolates. However, 20 out of 24 O6 strains carried CS2/CS3/CS21 genes, the major CF genes in ST171, even though they had different MLST types.

Based on the virulence factor investigation, NCCP15740 carried fewer virulence factors (59.4\%) than the average number of virulence factors $(62.0 \%)$ in the strains used in this study. In particular, $f \lg D$ and $f \lg E$ were not present in NCCP15740, but were detected in all of 
the reference strains. With respect to toxins, enterotoxinrelated genes (entA, entB, ent $C$, and entD) [28] were present in all of the O6 strains, including NCCP15740, whereas alpha-hemolysin-related genes ( $h l y A, h l y B, h l y C$, and $h l y D)$ [29] were only present in E. coli ATCC 25922. Alpha-hemolysin is a major virulence factor in ETEC, Shiga toxin-producing E. coli, and enteropathogenic strains and is thought to be acquired by horizontal gene transfer via conjugative plasmids [30].

Interestingly, none of the nonclassical virulence genes were detected in the NCCP15740 genome, and only three nonclassical virulence genes, i.e., etpA, irp2, and tia, were detected in other $\mathrm{O} 6$ strains. The $\mathrm{O} 6$ reference strains were isolated from patients in the USA, but the nonclassical virulence gene profiles were quite different from those of South American isolates. The eatA, irp2, and fyu $A$ genes were the most prevalent in Colombian and Chilean ETEC strains [25], but none of the genes were detected in $\mathrm{O} 6$ strains. In addition, the tia and leoA genes were less frequent in Bolivia [31], Chile [32], Guatemala, and Mexico [33], although 11 out of $24 \mathrm{O} 6$ strains had the tia gene.

\section{Future directions}

In summary, NCCP15740, representing the major type of ETEC in Korea, appeared to belong to the O6 serotype and ST4. Unlike other ST4 strains, NCCP15740 did not carry the CS21 gene. Moreover, the strain harbored fewer classical virulence factors than the O6 reference strains and did not contain any nonclassical virulence factors. These results provided important insights into the development of ETEC vaccine candidates. However, because the results were obtained from in silico analyses, experimental confirmation of the results is required.

\section{Additional file}

Additional file 1. Virulence factor profiles of NCCP15740 and reference strains

\section{Abbreviations}

BSR: BLAST score ratio; CDS: coding DNA sequences; CF: colonization factor; ETEC: enterotoxigenic Escherichia coli; LT: heat-labile toxin; STh: heat-stable toxin; MLST: multilocus sequence typing; NCCP: national culture collection for pathogens; RAST: rapid annotation using subsystem technology; str.: strain.

\section{Authors' contributions}

SHC and YSB planned and directed the project and interpreted the results. SHC drafted the manuscript. SJJ, SGR, JSP, CHK and WK interpreted the results. YHJ characterized the strain and prepared the genomic DNA. TK and SYC performed the gene annotation and comparative genomic analysis and wrote the manuscript. All authors read and approved the final manuscript

\section{Author details}

${ }^{1}$ Interdisciplinary Program in Bioinformatics, Seoul National University, 1 Gwanak-ro, Gwanak-gu, Seoul 151-742, Republic of Korea. ${ }^{2}$ Department of Biotechnology, Korea University, Anam-ro 145, Seongbuk-gu, Seoul 02841,
Republic of Korea. ${ }^{3}$ Department of Dental Hygiene, College of Medical Science, Konyang University, 158, Gwanjeodong-ro, Seo-gu, Daejeon-Metropolitan City 35365, Republic of Korea. ${ }^{4}$ Department of Emergency Medical Services, Sun Moon University, Asan, Chungcheongnam-do 31460, Republic of Korea. ${ }^{5}$ Fire Science Laboratory, National Fire Service Academy, Asan, Chungcheongnam-do 31555, Republic of Korea. ${ }^{6}$ Glycobiology Unit, Department of Biological Science, Sungkyunkwan University and Samsung Advanced Institute for Health Sciences and Technology (SAIHST), 2066 Seobu-ro, Suwon 16419, Republic of Korea. ${ }^{7}$ Division of Enteric Diseases, Center for Infectious Diseases, Korea National Institute of Health, Cheongju 363-951, Republic of Korea.

\section{Acknowledgements}

Not applicable.

\section{Competing interests}

The authors declare that they have no competing interests.

\section{Availability of data and materials}

Nucleotide sequence accession numbers: The whole-genome shotgun sequencing data have been deposited in DDBJ/EMBL/GenBank under the Accession Number MOED00000000.

\section{Consent for publication \\ Not applicable.}

\section{Ethics approval and consent to participate}

This research has been reviewed and approved by the Institutional Review Board of the Korea Centers for Disease Control and Prevention (Reference No.: 2013-12-04-P).

Written informed consent was obtained from all patients with diarrhea to participate the research.

\section{Funding}

This work was supported by a grant from the Marine Biotechnology Program (Genome Analysis of Marine Organisms and Development of Functional Applications) funded by the Ministry of Oceans and Fisheries and Sun Moon University.

\section{Publisher's Note}

Springer Nature remains neutral with regard to jurisdictional claims in published maps and institutional affiliations.

Received: 1 May 2017 Accepted: 8 September 2017

Published online: 20 September 2017

\section{References}

1. Robins-Browne RM, Hartland EL. Escherichia coli as a cause of diarrhea. J Gastroenterol Hepatol. 2002;17(4):467-75.

2. Wenneras C, Erling V. Prevalence of enterotoxigenic Escherichia coliassociated diarrhoea and carrier state in the developing world. J Health Popul Nutr. 2004;22(4):370-82.

3. WHO. Future directions for research on enterotoxigenic Escherichia coli vaccines for developing countries. Wkly Epidemiol Rec. 2006;81(11):97-104

4. Nataro JP, Kaper JB. Diarrheagenic Escherichia coli. Clin Microbiol Rev. 1998;11(1):142-201.

5. Qadri F, Svennerholm AM, Faruque AS, Sack RB. Enterotoxigenic Escherichia coli in developing countries: epidemiology, microbiology, clinical features, treatment, and prevention. Clin Microbiol Rev. 2005;18(3):465-83.

6. Gaastra W, Svennerholm AM. Colonization factors of human enterotoxigenic Escherichia coli (ETEC). Trends Microbiol. 1996;4(11):444-52.

7. Croxen MA, Law RJ, Scholz R, Keeney KM, Wlodarska M, Finlay BB. Recent advances in understanding enteric pathogenic Escherichia coli. Clin Microbiol Rev. 2013;26(4):822-80. 
8. Oh KH, Kim DW, Jung SM, Cho SH. Molecular characterization of Enterotoxigenic Escherichia coli strains isolated from diarrheal patients in Korea during 2003-2011. PLoS ONE. 2014;9(5):e96896.

9. Minogue TD, Daligault HA, Davenport KW, Bishop-Lilly KA, Broomall SM, Bruce DC, Chain PS, Chertkov O, Coyne SR, Freitas T, et al. Complete genome assembly of Escherichia coli ATCC 25922, a serotype O6 reference strain. Genome Announc. 2014;2(5):e00969.

10. Pattabiraman V, Bopp CA. Draft whole-genome sequences of 10 enterotoxigenic Escherichia coli serogroup O6 strains. Genome Announc. 2015;3(3):e00564.

11. Pattabiraman V, Bopp CA. Draft whole-genome sequences of 10 serogroup $\mathrm{O6}$ enterotoxigenic Escherichia coli strains. Genome Announc. 2014;2(6):e01274.

12. Madhavan TP, Steen JA, Hugenholtz P, Sakellaris H. Genome sequence of enterotoxigenic Escherichia coli strain B2C. Genome Announc. 2014;2(2):e00247.

13. Trees E, Strockbine N, Changayil S, Ranganathan S, Zhao K, Weil R, MacCannell D, Sabol A, Schmidtke A, Martin H, et al. Genome sequences of 228 Shiga toxin-producing Escherichia coli isolates and 12 isolates representing other diarrheagenic E. coli pathotypes. Genome Announc. 2014;2(4):e00718.

14. Li R, Zhu H, Ruan J, Qian W, Fang X, Shi Z, Li Y, Li S, Shan G, Kristiansen K, et al. De novo assembly of human genomes with massively parallel short read sequencing. Genome Res. 2010;20(2):265-72.

15. Aziz RK, Bartels D, Best AA, DeJongh M, DiszT, Edwards RA, Formsma K, Gerdes S, Glass EM, Kubal M, et al. The RAST server: rapid annotations using subsystems technology. BMC Genomics. 2008;9:75.

16. Joensen KG, Tetzschner AM, Iguchi A, Aarestrup FM, Scheutz F. Rapid and easy in silico serotyping of Escherichia coli isolates by use of wholegenome sequencing data. J Clin Microbiol. 2015;53(8):2410-26.

17. Wirth T, Falush D, Lan R, Colles F, Mensa P, Wieler LH, Karch H, Reeves PR, Maiden MC, Ochman H, et al. Sex and virulence in Escherichia coli: an evolutionary perspective. Mol Microbiol. 2006;60(5):1136-51.

18. Khan NH, Ahsan M, Yoshizawa S, Hosoya S, Yokota A, Kogure K. Multilocus sequence typing and phylogenetic analyses of Pseudomonas aeruginosa Isolates from the ocean. Appl Environ Microbiol. 2008;74(20):6194-205.

19. Glaeser SP, Kampfer P. Multilocus sequence analysis (MLSA) in prokaryotic taxonomy. Syst Appl Microbiol. 2015;38(4):237-45.

20. Angiuoli SV, Salzberg SL. Mugsy: fast multiple alignment of closely related whole genomes. Bioinformatics. 2011;27(3):334-42.

21. Price MN, Dehal PS, Arkin AP. FastTree: computing large minimum evolution trees with profiles instead of a distance matrix. Mol Biol Evol. 2009;26(7):1641-50.
22. Stamatakis A. Phylogenetic models of rate heterogeneity: a high performance computing perspective. 2006 IPDPS, 20th International Parallel and Distributed Processing Symposium 2006.

23. Chen L, Yang J, Yu J, Yao Z, Sun L, Shen Y, Jin Q. VFDB: a reference database for bacterial virulence factors. Nucleic Acids Res. 2005;33:D325-8.

24. Rasko DA, Myers GS, Ravel J. Visualization of comparative genomic analyses by BLAST score ratio. BMC Bioinformatics. 2005;6:2.

25. Guerra JA, Romero-Herazo YC, Arzuza O, Gomez-Duarte OG. Phenotypic and genotypic characterization of enterotoxigenic Escherichia coli clinical isolates from northern Colombia. South America. Biomed Res Int. 2014:2014:236260

26. Clemens J, Savarino S, Abu-Elyazeed R, Safwat M, Rao M, Wierzba T, Svennerholm AM, Holmgren J, Frenck R, Park E, et al. Development of pathogenicity-driven definitions of outcomes for a field trial of a killed oral vaccine against enterotoxigenic Escherichia coli in Egypt: application of an evidence-based method. J Infect Dis. 2004;189(12):2299-307.

27. Valvatne $H$, Steinsland $H$, Sommerfelt $H$. Clonal clustering and colonization factors among thermolabile and porcine thermostable enterotoxinproducing Escherichia coli. APMIS. 2002;110(9):665-72.

28. Tsen HY, Chen TR. Use of the polymerase chain reaction for specific detection of type A, D and E enterotoxigenic Staphylococcus aureus in foods. Appl Microbiol Biotechnol. 1992;37(5):685-90.

29. Burgos Y, Beutin L. Common origin of plasmid encoded alpha-hemolysin genes in Escherichia coli. BMC Microbiol. 2010;10:193.

30. Lim JY, Yoon J, Hovde CJ. A brief overview of Escherichia coli O157: H7 and its plasmid O157. J Microbiol Biotechnol. 2010;20(1):5-14.

31. Gonzales L, Sanchez S, Zambrana S, Iniguez V, Wiklund G, Svennerholm AM, Sjoling A. Molecular characterization of enterotoxigenic Escherichia coli isolates recovered from children with diarrhea during a 4-year period (2007-2010) in Bolivia. J Clin Microbiol. 2013;51(4):1219-25.

32. Del Canto F, Valenzuela P, Cantero L, Bronstein J, Blanco JE, Blanco J, Prado V, Levine M, Nataro J, Sommerfelt H, et al. Distribution of classical and nonclassical virulence genes in enterotoxigenic Escherichia coli isolates from Chilean children and tRNA gene screening for putative insertion sites for genomic islands. J Clin Microbiol. 2011;49(9):3198-203.

33. Turner SM, Chaudhuri RR, Jiang ZD, DuPont H, Gyles C, Penn CW, Pallen $\mathrm{MJ}$, Henderson IR. Phylogenetic comparisons reveal multiple acquisitions of the toxin genes by enterotoxigenic Escherichia coli strains of different evolutionary lineages. J Clin Microbiol. 2006;44(12):4528-36.

\section{Submit your next manuscript to BioMed Central and we will help you at every step:}

- We accept pre-submission inquiries

- Our selector tool helps you to find the most relevant journal

- We provide round the clock customer support

- Convenient online submission

- Thorough peer review

- Inclusion in PubMed and all major indexing services

- Maximum visibility for your research

Submit your manuscript at www.biomedcentral.com/submit 